\title{
Design of fiduciary markers for its implementation in the urban area
}

\section{SIGRADI2018 TECHNOPOLITICAS \\ xxii congresso da sociedade iberoamericana de gráfica digital 22th conference of the iberoamerican society of digital graphics 07|08|09|novembro|2018 iau usp | são carlos | sp br}

\author{
Bruno Perelli S. \\ Universidad de Chile| Chile | bperelli@uchilefau.cl \\ Pedro Soza R. \\ Universidad de Chile| Chile | psoza@uchilefau.cl
}

\begin{abstract}
The mediation by Augmented Reality, allows to add layers of virtual information to reality in natural and intuitive ways, guaranteeing interactions in real time and generating new perceptual stimuli not necessarily remitted exclusively to the field of vision. Interaction devices such as fiduciary markers are resources generally associated with RA experience, however these markers of paper-usually turn out to be ephemeral when implementation is done in open spaces and high traffic. The following work presents results in the analysis of materials that guarantee to be ideal for the permanent implementation of markers in pedestrian urban routes.
\end{abstract}

Keywords: Augmented Reality; Fiduciary Markers; Visual Impairment; Computer Vision.

\section{INTRODUCCIÓN}

El presente trabajo se sitúa en el diseño de un sistema alternativo de visión -actualmente en desarrollorecurriendo a algoritmos de visión por computador (CV), physical computing y realidad aumentada (AR).

Variados autores destacan el uso de AR dadas sus características de: Integrar imágenes virtuales en el mundo real (Maad, 2010); Su capacidad de proveer interfaces más naturales e intuitivas para la interacción con sistemas computacionales (Meiguins, Gonçalves Meiguins, Hernadez Almeida, de Moraes Lourenço y Vianna Pinheiro, 2010; Bieber y Raskar, 2005); Garantizando interacciones en tiempo real (Azuma,2001; Papagiannis, 2017) e integrando todo esto a nuestra percepción de la realidad (Greenfield, 2017).

La finalidad de esta propuesta de un nuevo "ojo" es la de registrar y generar estímulos visuales recurriendo a otros sentidos, de preferencia los sentidos auditivos y táctiles (Cattaneo y Vecchi, 2011), en situaciones en las que el órgano visual del ser humano presenta una discapacidad visual total o parcial. A estos estímulos alternativos generados a partir de las capacidades de neuroplasticidad del cerebro- se les denominan compensaciones sensoriales (Cattaneo y Vecchi, 2011) o substituciones sensoriales (Costandi, 2016).

El sistema detecta y obtiene información a través de marcadores fiduciarios —comúnmente conocidos como QR's- Estos marcadores encierran, en su diseño reticular de 12x12 módulos, valores binarios entre 0 y 1 traducidos en el contraste de color entre blanco y negro, los cuales generalmente se imprimen para su implementación (Figura 1).

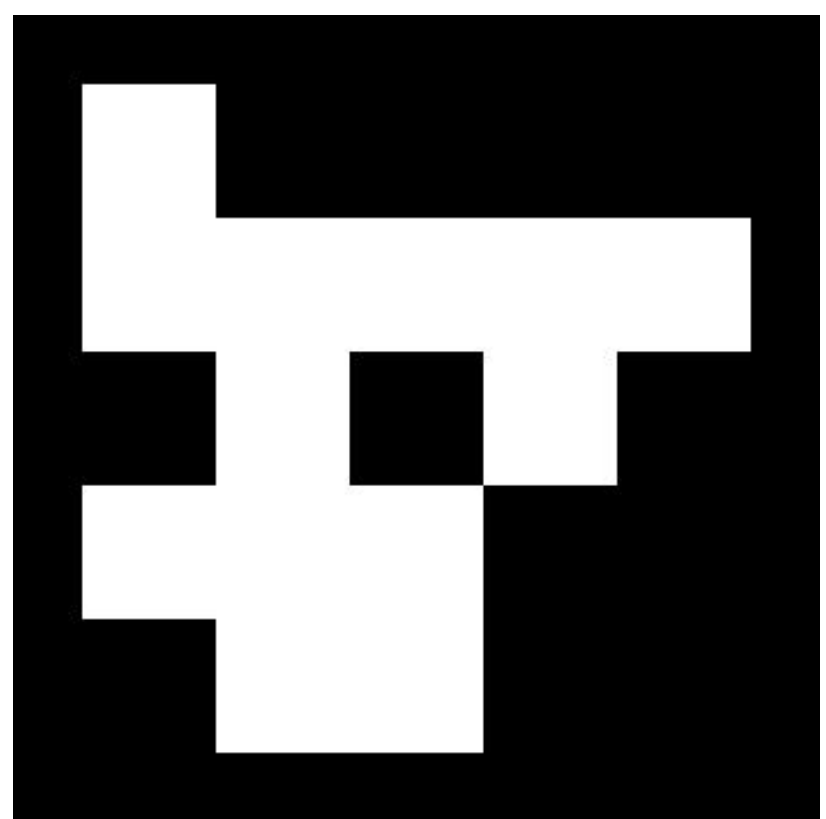

Figura 1: Marcador fiduciario compuesto por una matriz de $12 \times 12$ módulos los cuales contienen información binaria. Fuente: autores.

Las combinatorias de estos módulos ofrecen diseños de patrones que asocian mediante AR puntos en el espacio en un sistema de coordenadas cartesianas $(x, y)$. Si se llegan a detectar dos marcadores con el mismo diseño, se obtendrá su ID numérico correspondiente a ese diseño además de las coordenadas espaciales $\mathrm{x}, \mathrm{y}$ del punto centro de cada marcador uniéndolos a través de una línea virtual. Por lo tanto: los marcadores se disponen de tal manera que un diseño dibujará el borde de un camino establecido, mientras un segundo diseño hará lo mismo al otro extremo del recorrido (Figura 2). 


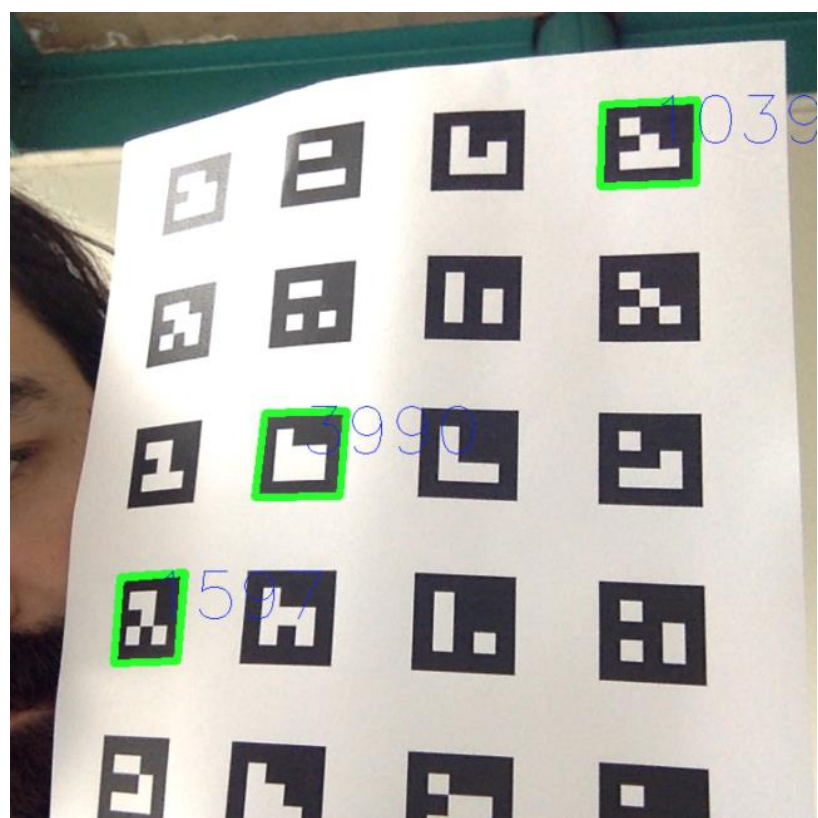

Figura 2: Marcadores fiduciarios entregando su identificador numérico (OpenCv+ARUCO). Fuente: autores.

Finalmente, operadores matemáticos - Canny86 y Hough Line- utilizados en visión por computador (CV) simplifican la imagen capturada y calculan el punto centro del recorrido dado por sus extremos los cuales pueden variar dependiendo de las distancias entre juegos de marcadores.

A partir de esto es que se han diseñado - en una fase preliminar- soluciones de software y Hardware especialmente desarrollado para traducir la data detectada (estímulos visuales resultantes de AR) en respuestas hápticas que favorecen el desplazamiento pedestre informando al usuario de su ubicación (izquierda, centro y derecha) en el recorrido a través de las ya mencionadas compensaciones sensoriales.

En esta segunda iteración del proyecto, han surgido nuevos desafíos para el equipo investigador: Por una parte, las condiciones lumínicas del entorno no siempre garantizan la lectura correcta de los marcadores. Dadas las características de operadores como Canny 86 se requiere simplificar la imagen capturada a una escala de grises y posteriormente aplicar un desenfoque Gaussiano para poder detectar efectivamente los bordes del entorno.

Por otra parte, los primeros trabajos con usuarios demostraron que dar tres opciones de ubicación (laterales y centro) para la toma de decisiones genera confusión cuando se trata de espacios muy amplios o reducidos. Tal como evidencia Jacobson (2013), la precisión de la ubicación exacta puede significar la diferencia entre estar orientado y perderse.

Finalmente, la materialidad de los marcadores, generalmente impresos en papel, lo vuelve un objeto fácil de reemplazar, pero poco operativo dada su limitada duración y resistencia.

El siguiente trabajo se remite a tres frentes de iteración en esta segunda etapa de este proyecto: En primer lugar, la elección de materiales alternativos al comúnmente utilizado papel para el diseño de marcadores fiduciarios mirados bajo dos grandes parámetros: El primero, es garantizar la correcta lectura e interpretación de estos por el software de AR. El segundo, es la de utilizar materiales que permitan su implementación duradera en espacios urbanos para desplazamiento pedestre.

En segundo lugar, resolver problemas con respecto a la detección de dichos marcadores bajo diferentes iluminantes basados en diversos horarios del día. De esta manera se hace hincapié en mejorar tanto software como hardware en cuanto a detección, transducción de datos y elaboración de respuestas.

El último frente, corresponde a refinar las respuestas compensatorias en el usuario de forma tal de entregar con mayor precisión la ubicación del sujeto al interior de los recorridos virtuales creados por los marcadores.

\section{PROCEDIMIENTOS METODOLÓGICOS}

La Realidad Aumentada debe ser considerada una forma de mediación en la cual podemos integrar imágenes virtuales a la realidad de modo tal que son capaces de extender nuestra percepción dada la interacción con elementos digitales (Greenfield, 2017; Papagiannis, 2017; Maad, 2005). Es efectivamente esta mediación la que ha sido considerada en el desarrollo de esta experiencia de compensación sensorial (Perelli y Soza, 2017), frente a la que los estímulos visuales no pasan por el ojo sino más bien son capturados a través de una cámara, transducidos por un computador, el cual diseña una nueva respuesta a través de motores vibratorios que estimulan la piel del sujeto indicando su posición al interior de un recorrido.

\section{CONFECCIÓN DE NUEVOS MARCADORES}

Durante el desarrollo de la iteración anterior, la utilización de marcadores fiduciarios impresos en papel demostró ser muy efectivo para la lectura y posterior detección de puntos en el espacio, sin embargo, todas las mediciones de dicha etapa siempre fueron desarrollados en espacios controlados dentro de los cuales las condiciones ambientales son mucho más controlables.

De esta forma, la iluminación, las condiciones climáticas, el tiempo y todo tipo de factor que pudiese afectar a los marcadores -y por consiguiente la correcta detección y lectura- garantizaban que, en esta etapa de experimentación, la búsqueda de materiales definitivos hubiese sido considerada, pero no como una prioridad imperante.

No obstante, nos encontramos en una segunda instancia donde ajustes definitivos son imperativos para la mejora de la experiencia de los usuarios. Basados en dicha premisa, es que el equipo de investigación centró sus esfuerzos en levantar un catálogo de posibles materiales aptos para la confección de marcadores que conserven las características originales tanto de diseño como función, pero que garanticen una mayor resistencia al momento de ser utilizados en espacios urbanos.

Las primeras pruebas se remitieron a probar materiales que generalmente se encuentran disponibles para el prototipado en la mayoría de los FabLabs: MDF y Acrílico. 
Tomando los diseños originales desarrollados para los marcadores impresos en papel, se optó por grabar ambos materiales con máquinas de corte láser con la finalidad de generar las zonas de contraste característicos de los marcadores fiduciarios los cuales encierran en su disposición cuadrantes con valores binarios: 1 (Blanco, Sí, Verdadero) y 0 (Negro, No, Falso).

Al realizar pruebas de la legibilidad de los marcadores frente al computador, inmediatamente surgió el primer problema de lectura: El operador Canny86. Este algoritmo, fue diseñado especialmente para capturar los bordes de la información visual presentada en las imágenes descartando la información de color (Perelli y Soza, 2017).

Canny inmediatamente detectó dobles líneas dadas por el pequeño relieve que dejó el grabado por láser, además de considerar las formas presentes en las texturas del MDF y los brillos y reflejos que se generan en la superficie del acrílico.

Es a partir de estos resultados preliminares que el equipo decide cambiar la elección de materiales y se centra en la búsqueda de materiales utilizados generalmente en la vía pública. Se debió tener especial preocupación en buscar materiales que garantizaran una lectura fluida de la información asociada al marcador (Líneas Rectas y contraste entre zonas). Para lograr esto, se diseñó un pequeño script que descompone el algoritmo Canny86 permitiendo un análisis parametrizado de cada una de sus operaciones de forma tal de encontrar no solo el material adecuado, sino, además, de obtener valores precisos de calibración para la cámara. Adicionalmente, el script utiliza Hough Lines, operador encargado de encontrar líneas rectas presentes en imágenes procesadas con Canny (Figura 3).

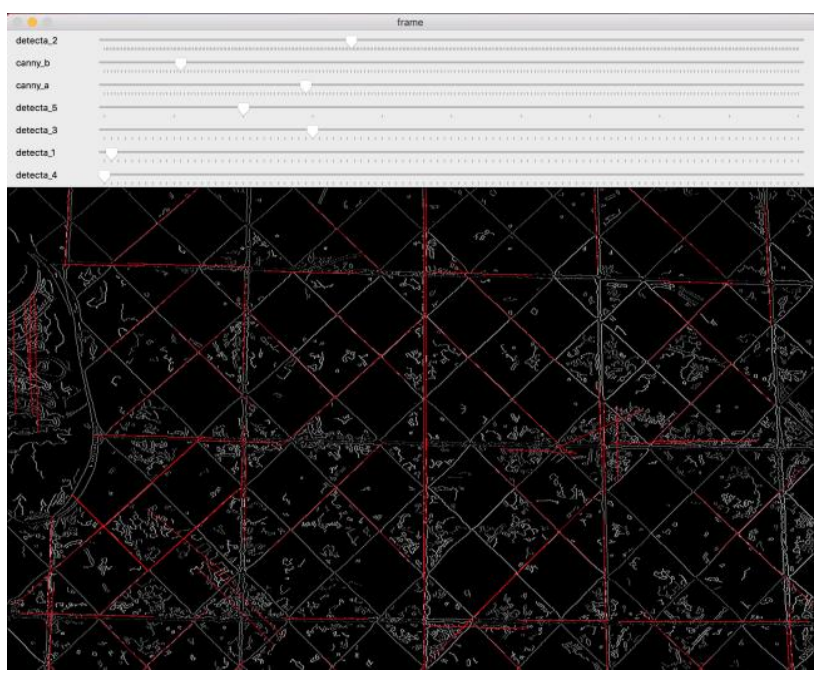

Figura 3: Script de parametrización de Canny. Fuente: autores.

El equipo comenzó a capturar imágenes de veredas con alto tránsito pedestre, decidiendo evaluar bajo la mirada del software una serie de baldosas: Un primer grupo corresponde a las llamadas Baldosas Pulidas, las cuales se utilizan con mayor frecuencia en las calles de la ciudad de Santiago de Chile. Un segundo grupo corresponde a las denominadas Baldosas Hidráulicas, las cuales ofrecen mayor variedad de diseños y colores, pero que generalmente se utilizan en menor medida en espacios exteriores privilegiados los espacios interiores.

Un elemento común y distintivo de estas baldosas es que son diseñadas de manera muy similar a los marcadores de papel utilizados con anterioridad siendo estas confeccionadas a partir de una retícula modular que puede variar en su número y disposición. La diferencia radica en que el primer grupo de Baldosas Pulidas tiene pequeños surcos lo cual genera ciertos relieves en su superficie. Por otra parte, el segundo grupo de Baldosas Hidráulicas son planas, permite mayor variedad de colores -lo que se traduce en mayor contraste entre zonas- y son de terminación mate evitando generar brillos en su superficie.
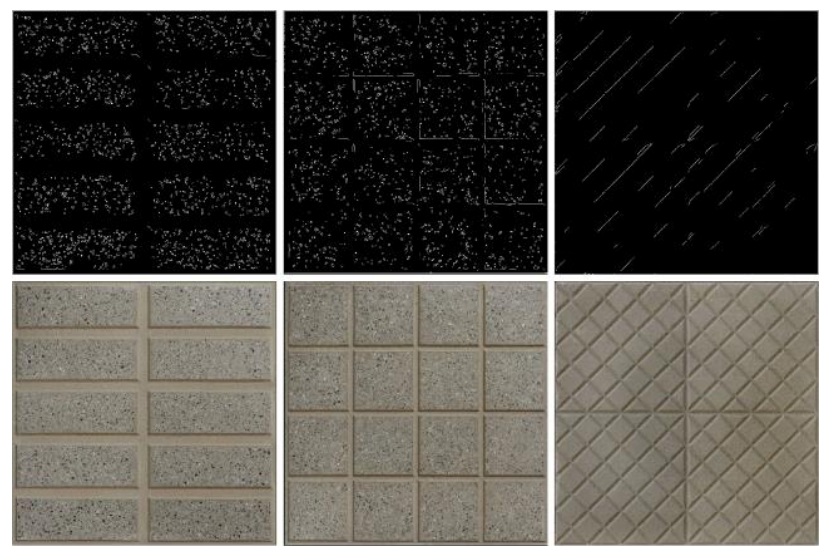

Figura 4: Análisis de Baldosas Pulidas. Fuente: autores.

Como se puede apreciar en la Figura 4, el análisis del primer grupo de Baldosas deja a la vista problemas en los mismos frentes que se advirtieron al momento de evaluar materiales como MDF y Acrílico: Las características del material en su composición generan ruidos visuales que rompen con el efecto deseado de leer contrastes entre negro y blanco. En segundo lugar, los surcos antes descritos generan valles en la superficie los cuales son interpretados por Canny como líneas independientes lo cual en el diseño final procesado se lee como dobles líneas.
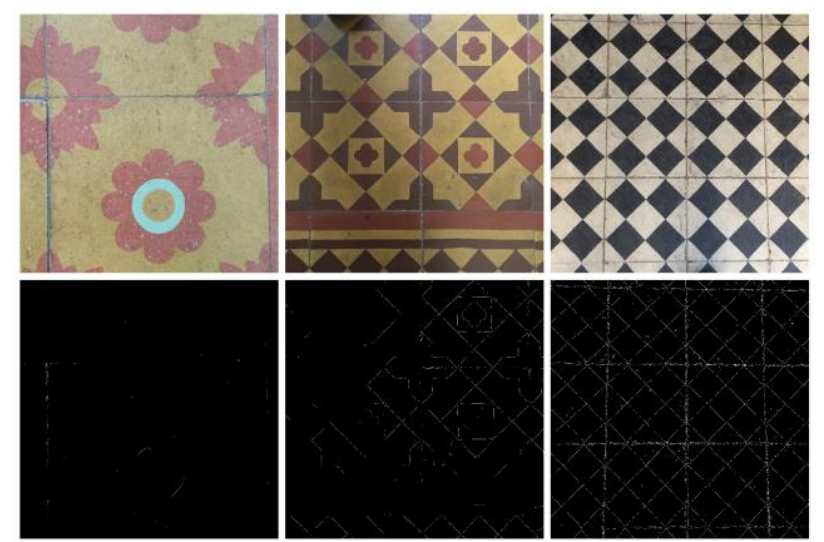

Figura 5: Análisis de Baldosas Hidráulicas bajo Canny86. Des izquierda a Derecha Muestras A, B y C. Fuente: autores.

Por otra parte, la serie de muestras correspondientes a las Baldosas Hidráulicas (Figura 5), arrojaron resultados distintos siendo estos los que generaron una lectura más 
fluida basado en contraste, líneas rectas (Fig. 5 C) y limpieza en la lectura.

En una instancia posterior, la muestra elegida, pasa a ser procesada por el software de parametrización de Canny y la detección de líneas rectas mediante el uso de Hough Lines. Los resultados entregados confirman una correcta lectura de líneas rectas (Figura 6), siendo las baldosas Hidráulicas con color en módulos de color negro y blanco aptas para la implementación del sistema. Sin embargo, hay que considerar que el borde original del marcador cumple dos funciones: Por una parte, es el encargado de delimitar el marcador, pero por otro también alberga data relevante para la identificación de este. Esto implica agregar un borde extra en la confección final del prototipo de baldosa.

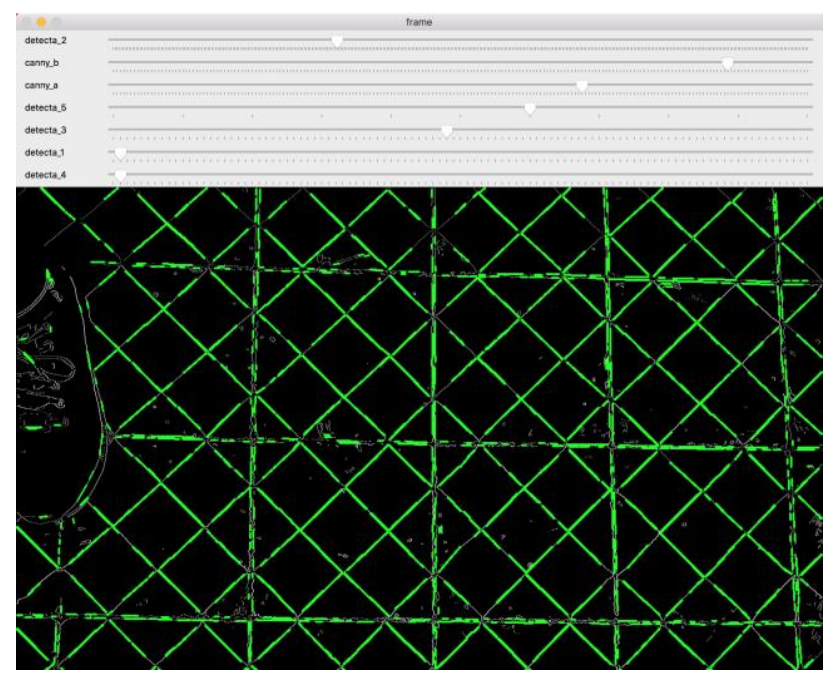

Figura 5: Análisis de Baldosas Hidráulicas bajo Hough Lines. Las líneas Verdes se consideran líneas rectas constantes. Fuente: autores.

\section{ACTUALIZACIONES DEL SOFTWARE Y HARDWARE}

Esta etapa recogió una serie de problemas surgidos en la iteración anterior del proyecto relacionado con una lectura fidedigna y constante de los marcadores en todo momento.

Condiciones lumínicas, climáticas y horario-temporales, demostraron afectar en la detección e interpretación de los marcadores en experiencias pasadas. Basado en esto es que el equipo ha considerado mejoras mayores tanto en el software de detección como en el hardware de captura y elaboración de las respuestas compensadas. Solo los factores climáticos se consideran solucionados por la confección de la Baldosa Fiduciaria descrita en el método anterior.

En cuanto a las actualizaciones de Hardware, diferencias significativas se han implementado desde el desarrollo del prototipo inicial, tal como advierte Kiyokawa (2007), no existe un HMD (o Head Mounted Display) perfecto, variados son los intentos de implementar un HMD eficiente y poco invasivo. No obstante, esta segunda versión del prototipo considera integrar soluciones que respondan a los factores anteriormente mencionados (Horarios-Temporales y Lumínicos).
Esto se traduce en la integración de una cámara infrarroja (IR) a la ya existente cámara RGB del prototipo inicial (Figura 8). La cámara IR garantiza una captura en espacios de oscuridad total o parcial dado que recurre a iluminación de LEDs infrarrojos montados a su alrededor. Esta mejora demandó entregarle al software recursos de ubicación temporales y geográficos dado que dependiendo del lugar, fecha o estación del año el prototipo debe decidir por una de las dos cámaras. Basado en esto se cargan librerías de Geocodificación las cuales consultan fecha, ubicación y a partir de eso establece una variable llamada AHORA.

Finalmente, se establecen las variables AMANECER y $A T A R D E C E R$ las cuales varían según la zona horaria y la estación del año, por lo tanto, los valores de AMANECER y ATARDECER serán completamente distintos día a día, semana a semana, mes a mes y año a año. Se debe considerar que Chile utiliza dos zonas horarias distintas durante estaciones Estivales e Invernales. De esta manera:

\section{$($ AMANECER $<A H O R A<A T A R D E C E R)=D I I A=$ Cámara RGB}

\section{$($ AMANECER $<$ ATARDECER $<$ AHORA $)=N O C H E=$ Cámara IR}

La elección de cámaras resultó de forma exitosa generando lecturas fluidas en espacios de alta luminosidad como en espacios de luminosidad reducida, no generando problemas al interpretar los marcadores.

Una segunda mejora de Software y Hardware con respecto a la versión anterior tiene relación con la elaboración de la Respuesta compensada. Como ya se advirtió el prototipo uno consideraba tres estímulos vibratorios a partir de motores que indican el extremo izquierdo, derecho o el centro del camino.

Los marcadores dibujan a través de AR los extremos del camino, pero además entregan el valor de ángulo de bisectriz obtenido por la perspectiva y los puntos centros entre ambos extremos del recorrido. El software interpreta esta data y establecía —originalmente- rangos entre 0 , 45 y 90 grados. Esta diferencia entre rangos se consideró satisfactoria en una primera instancia, sin embargo, pruebas con usuarios demostraron ser valores demasiado amplios al traducirlos a distancias. Es por esto que, en esta nueva versión, el equipo optó por aumentar los estímulos vibratorios referenciales de los tres originales a siete estímulos diferentes generando así transiciones mucho más especificas entre los puntos del recorrido.

El resultado de esto es que ahora el usuario se encuentra con tres niveles de ubicación para el lado izquierdo, uno para el centro y otros tres niveles para el lado derecho del camino variando los intervalos de $45^{\circ}$ (cuarenta y cinco grados) en el prototipo uno a $15^{\circ}$ (quince grados) en el nuevo prototipo.

\section{RESULTADOS Y DISCUSIÓN}

La integración de las mejoras de Hardware y Software ofrece a los usuarios mayor precisión al momento de detectar marcadores en condiciones lumínicas variables dada la implementación de tecnología IR. 
Desde el punto de vista del HMD, se optó por integrar las nuevas mejoras en el marco de unos lentes para realidad virtual los cuales se modificaron para albergar los nuevos dispositivos de captura y construcción de respuestas compensadas traducidas en siete posibles estímulos vibratorios. No se descartan nuevos cambios a partir de las nuevas pruebas de usuarios.

Por otra parte, la evaluación de materiales tanto de FabLab (MDF y Acrílico), como las baldosas evaluadas durante el desarrollo de esta investigación, permitieron obtener resultados que por una parte entregan información relevante al momento de establecer los parámetros de medición de lectura cuando los posibles marcadores no están confeccionados en materiales perecederos como el papel.

Sin embargo, dadas las nuevas mejoras en Hardware y Software con respecto a las tecnologías IR de colores no perceptibles por el ojo humano, es que no se descartan realizar nuevas indagatorias similares a las experiencias en marcadores fiduciarios propuestos por Wang, Liu, Chang y Chen (2008).

Cabe señalar que, a la fecha, aún faltan nuevos antecedentes en cuanto a pruebas de legibilidad, detección y reconocimiento de patrones fiduciarios en los prototipos de baldosas dado que, a la fecha de finalización de este escrito, los prototipos se encuentran en proceso de producción para la realización de los test finales.

Se espera que se mantenga una concordancia con respecto a los análisis preliminares en baldosas hidráulicas en color negro y blanco tal y como fue demostrado durante los procesos de medición de esta investigación.

Con respecto a la utilización de AR, es interesante destacar que — tal como alude Greenfield (2017) — una técnica de mediación como esta nos permite Diseñar nuevas capaz de información relevantes al momento de percibir el espacio, no solo traducido en elementos virtuales visibles, sino que son capaces de construir nuevos dialectos visuales. Esto supone no solamente mejoras en la calidad de vida de personas con discapacidad visual en distintos grados, tampoco solo se remite a la implementación de nuevas formas de ver el entorno a partir de objetos capaces de ser legibles bajo parámetros tecnológicos como es la propuesta de Baldosas Fiduciarias aquí propuestas, sino que también nos hace validar la idea planteadas por autores como Ings (2008), el cual establece que en cuanto a la visión, no es necesaria la presencia de un ojo para poder ver el entorno.

\section{AGRADECIMENTOS}

Esta investigación contó con el apoyo del concurso de investigación FAU 2.0 2016, de la facultad de arquitectura y urbanismo de la Universidad de Chile.

\section{REFERENCIAS}

Azuma, R., Baillot, Y., Behringer, R., Feiner, S., Julier, S., \& Maclntyre, B. (2001). Recent advances in augmented reality.
IEEE Computer Graphics and Applications, 21(6), 34-47. http://doi.org/10.1109/38.963459

Bimber, O., \& Raskar, R. (2005). Spatial Augmented Reality: Merging Real and Virtual Worlds. Wellesley, Massachusetts: A K Peters.

Cattaneo, Z., \& Vecchi, T. (2011). Blind Vision: The Neuroscience of Visual Impairment. Cambridge, Massachusetts: The MIT Press.

Costandi, M. (2016). NEUROPLASTICITY. Cambridge, Massachusetts: The MIT Press Cambridge.

Greenfield, A. (2017). Augmented reality: An interactive overlay on the world. En Radical Technologies:The Design of Everyday Life (1a ed.). Brooklyn, NY: Verso.

Ings, S. (2008). A Natural History of Seeing: The Art and Science of Vision (1 edition). W. W. Norton \& Company.

Jacobson, W. H. (2013). Orientation and Mobility. In R. Manduchi \& S. Kurniawan (Eds.), Assistive Technology for Blindness and Low Vision (p. 29). Boca Raton, FL: CRC Press.

Kiyokawa, K. (2007). An Introduction to Head Mounted Displays for Augmented Reality. En M. Haller, M. Billinghurst, \& B. H. Thomas (Eds.), Emerging Technologies of Augmented Reality: Interfaces and Design (1a ed., pp. 43-63). IDEA Group Publishing

Maad, S. (Ed.). (2010). Augmented Reality (1a ed.). Vukovar, Croatia: Intech.

Meiguins, B. S., Gonçalves Meiguins, A. S., Hernadez Almeida, L., de Moraes Lourenço, R. A., \& Vianna Pinheiro, S. C. (2010). Coordinated and Multiple Data Views in Augmented Reality Environment. En S. Maad (Ed.), Augmented Reality (1a ed., pp. 1-13). Vukovar, Croatia: Intech.

Papagiannis, H. (2017). Augmented Human: How Technology Is Shaping the New Reality. (J. Bleiel \& S. Conant, Eds.) (First Edit). O'Reilly Media.

Peddie, J. (2017). Augmented Reality. Cham: Springer International Publishing. http://doi.org/10.1007/978-3-31954502-8

Perelli Soto, B., \& Soza Ruiz, P. (2017). Soporte Visual Asistivo para la Detección de Formas Matemáticas en el entorno. En Blucher Design Proceedings (pp. 579-584). São Paulo: Editora Blucher. http://doi.org/10.5151/sigradi2017-089

Wang, H.-C., Liu, W.-H., Chang, C.-L., \& Chen, Y.-H. (2008). Design of Halftone-Based AR Markers under Infrared Detection. En 2008 International Conference on Computer Science and Software Engineering (pp. 97-100). IEEE. http://doi.org/10.1109/CSSE.2008.1391
5 